\title{
Targeted genomic enrichment and sequencing of CyHV-3 from carp tissues confirms low nucleotide diversity and mixed genotype infections
}

Saliha Hammoumi ${ }^{1}$, Tatiana Vallaeys ${ }^{2}$, Ayi Santika ${ }^{3}$, Philippe Leleux ${ }^{4}$, Ewa Borzym ${ }^{5}$, Christophe Klopp ${ }^{4}$, Jean-Christophe Avarre ${ }^{\text {Corresp. } 1}$

1 Institut des Sciences de l'Evolution de Montpellier, Institut de Recherche pour le Développement, Montpellier, France

2 Université de Montpellier, Montpellier, France

3 Main Center for Freshwater Aquaculture Development, Sukabumi, Indonesia

4 Plate-forme Genotoul Bioinfo, UR875 Biométrie et Intelligence Artificielle, Institut National de la Recherche Agronomique, Castanet-Tolosan, France

5 Department of Fish Diseases, National Veterinary Research Institute, Pulawy, Poland

Corresponding Author: Jean-Christophe Avarre

Email address: jean-christophe.avarre@ird.fr

Koi herpesvirus disease (KHVD) is an emerging disease that causes mass mortality in koi and common carp, Cyprinus carpio L. Its causative agent is Cyprinid herpesvirus 3 (CyHV3), also known as koi herpesvirus (KHV). Although data on the pathogenesis of this deadly virus is relatively abundant in the literature, still little is known about its genomic diversity and about the molecular mechanisms that lead to such a high virulence. In this context, we developed a new strategy for sequencing full-length CyHV-3 genomes directly from infected fish tissues. Total genomic DNA extracted from carp gill tissue was specifically enriched with CyHV-3 sequences through hybridization to a set of nearly 2 million overlapping probes designed to cover the entire genome length, using KHV-J sequence (GenBank accession number AP008984) as reference. Applied to 7 CyHV-3 specimens from Poland and Indonesia, this targeted genomic enrichment enabled recovery of the full genomes with $>99.9 \%$ reference coverage. The enrichment rate was directly correlated to the estimated number of viral copies contained in the DNA extracts used for library preparation, which varied between $\sim 5000$ to $\sim 2 \times 10^{7}$. The average sequencing depth was $>200$ for all samples, thus allowing the search for variants with high confidence. Sequence analyses highlighted a significant proportion of intra-specimen sequence heterogeneity, suggesting the presence of mixed infections in all investigated fish. They also showed that inter-specimen genetic diversity at the genome scale was very low (> $99.95 \%$ of sequence identity). By enabling full genome comparisons directly from infected fish tissues, this new method will be valuable to trace outbreaks rapidly and at a reasonable cost, and in turn to understand the transmission routes of CyHV-3. 
1 Targeted genomic enrichment and sequencing of $\mathrm{CyHV}-3$ from carp

2

3

4

5

6

7

8

9

10

11

12

13

14

15

16

17

18

19

20

21

22

23

24

25

26

Saliha Hammoumi ${ }^{1}$, Tatiana Vallaeys ${ }^{2}$, Ayi Santika ${ }^{3}$, Philippe Leleux ${ }^{4}$, Ewa Borzym ${ }^{5}$, Christophe Klopp ${ }^{4}$, Jean-Christophe Avarre ${ }^{1}$ infections

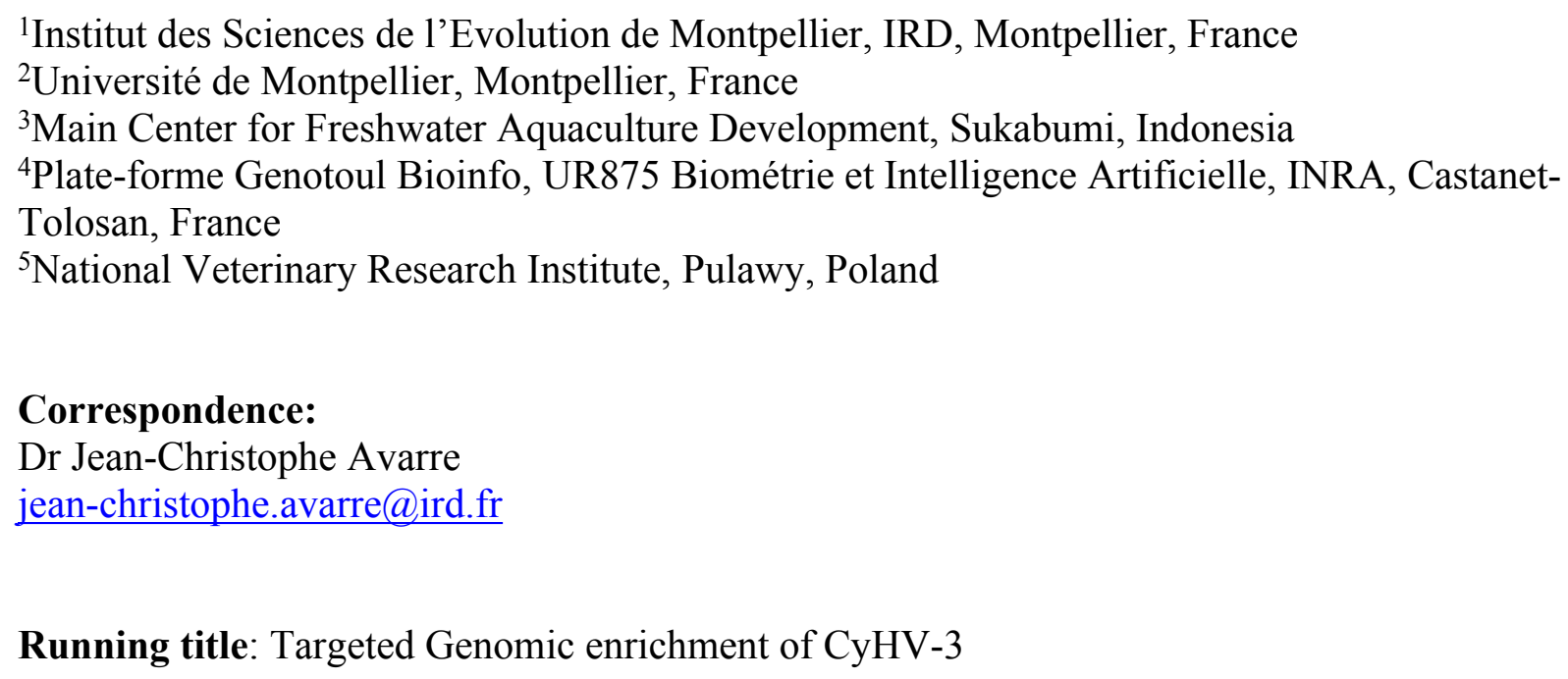

Running title: Targeted Genomic enrichment of CyHV-3 


\begin{abstract}
27 Abstract
28

29

30

31

32

33

34

35

Koi herpesvirus disease (KHVD) is an emerging disease that causes mass mortality in koi and common carp, Cyprinus carpio L. Its causative agent is Cyprinid herpesvirus 3 (CyHV-3), also known as koi herpesvirus (KHV). Although data on the pathogenesis of this deadly virus is relatively abundant in the literature, still little is known about its genomic diversity and about the molecular mechanisms that lead to such a high virulence. In this context, we developed a new strategy for sequencing full-length CyHV-3 genomes directly from infected fish tissues. Total genomic DNA extracted from carp gill tissue was specifically enriched with CyHV-3 sequences through hybridization to a set of nearly 2 million overlapping probes designed to cover the entire genome length, using KHV-J sequence (GenBank accession number AP008984) as reference. Applied to 7 CyHV-3 specimens from Poland and Indonesia, this targeted genomic enrichment enabled recovery of the full genomes with $>99.9 \%$ reference coverage. The enrichment rate was directly correlated to the estimated number of viral copies contained in the DNA extracts used for library preparation, which varied between $\sim 5000$ to $\sim 2 \times 10^{7}$. The average sequencing depth was $>200$ for all samples, thus allowing the search for variants with high confidence. Sequence analyses highlighted a significant proportion of intra-specimen sequence heterogeneity, suggesting the presence of mixed infections in all investigated fish. They also showed that interspecimen genetic diversity at the genome scale was very low ( $>99.95 \%$ of sequence identity). By enabling full genome comparisons directly from infected fish tissues, this new method will be valuable to trace outbreaks rapidly and at a reasonable cost, and in turn to understand the transmission routes of CyHV-3.
\end{abstract}




\section{Introduction}

Koi herpesvirus disease (KHVD) is an emerging disease that causes mass mortalities in koi and common carp, Cyprinus carpio L. Since the first report in the late 1990s (Hedrick et al. 2000), the disease has spread to many countries worldwide (Pokorova et al. 2005). The causative agent, cyprinid herpesvirus 3 (CyHV-3), is a large double-stranded DNA virus belonging to the Alloherpesviridae family, genus Cyprinivirus (Davison 2010; King et al. 2011; Waltzek et al. 2005). Twelve alloherpesviruses have been described to date: two of them infect amphibians while the ten remaining infect various fish species, including eel, salmon, catfish, goldfish and sturgeon (Waltzek et al. 2009).

Although the pathogenesis of this virus is well understood and well described in the literature, little is known about the genomic diversity and evolution. Sequencing of three complete CyHV-3 genomes of isolates originating from the USA (U), Israel (I) and Japan (J) (Aoki et al. 2007) revealed that sequences were highly similar $(>99.9 \%)$, which was consistent with a scenario of a unique virus that spread worldwide. Meanwhile, accurate genomic comparisons highlighted two main genetic lineages formed by the U/I and J strains. The existence of two lineages was further confirmed on a larger set of European and Asian isolates by using PCR-based molecular markers targeting various regions of the genome (Bigarré et al. 2009; Kurita et al. 2009). These two lineages were designated as European and Asian. Finally, the use of variable number of tandem repeats (VNTR) revealed a whole range of variants associated with both European and Asian lineages (Avarre et al. 2011). It was also demonstrated that genetically distinct strains of CyHV3 may co-occur in a single enzootic area, and that carp were frequently infected with a mix of genotypes (Avarre et al. 2012; Sunarto et al. 2011). Although viruses evolve more rapidly than their hosts, the differentiation ability of CyHV-3 remains mostly unknown, and consequently, it is difficult to predict whether the presence of distinct genotypes within a single host results from distinct infection events, from the evolution of a unique strain, or from both. Thus, characterizing these genetic variants at the genome level is critical to better understand the ecological and evolutionary significance of such mixed infections, especially with regards to the virulence and the modes of propagation of the virus.

In this context, the objective of the present work was to develop a method enabling the sequencing of CyHV-3 full-length genomes directly from their host. Targeted genomic enrichment (TGE), or targeted sequence capture prior to sequencing, has been described for a large panel of applications (Mertes et al. 2011). In spite of its great potential in virology, the application of this approach has remained limited since its first description and successful application to human herpesviruses (Depledge et al. 2011). Using a set of probes designed to specifically capture CyHV-3 sequences, seven genomes were fully sequenced from carp gill tissue samples collected during several outbreaks. In order to reduce the cost associated with TGE, the influence of pre-capture multiplexing on the ability to recover full genomes and to discover mutations in CyHV-3 with high confidence was also evaluated.

\section{Material and Methods}

\section{CyHV-3 specimens and DNA extraction}

Seven CyHV-3 specimens were used in the present study. They consisted of gill tissues collected from infected carps and stored in $96 \% \mathrm{EtOH}$ at $-20^{\circ} \mathrm{C}$. Six of them (J1_101110, CB4_181110, PP3_070411, I_10-3, I_09-2i3, J2_101110) were obtained from moribund cultured common 
97 carps collected in Indonesia (Sukabumi district, West Java), during different outbreaks (Table 1), 98 whereas the seventh (PoB3) was obtained from a common carp collected during an outbreak in 99 Poland. Total genomic DNA was extracted from a small gill fragment with the DNAzol kit (Life technologies) and the Wizard Genomic DNA purification kit (Promega) for the Indonesian and Polish specimens, respectively, following the appropriate protocols provided by the manufacturers. DNA concentration was measured by fluorometry (Qbit, Life Technologies), and its integrity was verified by capillary electrophoresis (Bioanalyzer 2100, Agilent). The viral DNA concentration was determined in each sample by real-time PCR, using the same method as previously described (Avarre et al. 2011; Yuasa et al. 2005).

\section{CyHV-3 genome enrichment and sequencing}

About $2 \mu \mathrm{g}$ of total DNA were used to establish the genomic libraries of each sample, using the TruSeq DNA sample preparation kit (Illumina). DNA was sonicated using a Bioruptor device (Diagenode). The average insert size used for sequencing was $\sim 300 \mathrm{bp}$. After adapter ligation using the Truseq kit, libraries were subjected to a viral-specific sequence capture using the SeqCap EZ library SR technology (Roche-Nimblegen). A large set of 50-105mer CyHV-3 specific probes was designed (Roche-Nimblegen) to theoretically capture all DNA sharing more than $90 \%$ identity with the published CyHV-3 genome of strain J (GenBank \#AP008984). This probe set (Roche Diagnostics design OID 41307, IRN 4000012980) is available upon demand.

A pre-capture ligation-mediated PCR (LM-PCR) consisting of 8 cycles of PCR was first performed according to the manufacturer's instructions (Illumina). After purification using Agencourt Ampure XP beads (Beckman Coulter), the amplified products were hybridized with the CyHV-3 probes at $47^{\circ} \mathrm{C}$ for 64 hours. In 4-plex assays, $250 \mathrm{ng}$ of four amplified products were pooled prior to hybridization. At the end of incubation, hybridized DNA was recovered using Nimblegen capture beads. Then 18 cycles of a post-capture LM-PCR were carried out on each captured DNA sample. DNA was again purified using Ampure beads, and its concentration was measured using a spectrophotometer (Nanodrop 1000) while its integrity was verified using a Bioanalyzer 2100 (Agilent).

In view of reducing the experimental costs, we compared the efficiency of a pre-capture multiplexing (4-plex) design to that of a simplex one, using specimen PoB3 as "control" because this sample displayed the lowest viral load (Table 1). Each library had initially been ligated with a unique Illumina adaptor, and two 4-plex assays were designed according to the estimated viral loads: the first included PoB3 and 3 Indonesian specimens with high viral loads $\left(>10^{6}\right.$ genome copies) whereas the second comprised PoB3 and the 3 remaining Indonesian specimens that displayed lower loads $\left(\leq 1.5 \times 10^{5}\right.$ genome copies). In parallel, PoB3 was also subjected to a double-capture protocol, which consisted of two overnight hybridizations at $47^{\circ} \mathrm{C}$ followed by a non-specific DNA elution using capture beads. All sample combinations were sequenced on a single lane of a HiSeq2000 platform (Illumina) in a paired-end (100 bases) format, at Montpellier Genomix (Montpellier, France).

\section{Genome assembly/mapping and detection of variations}

Raw sequences (fastq files) were stored in the public Sequence Read Archive (SRA) repository under the SRP study accession SRP05976 as well as in the NG6 repository (Mariette et al. 2012) of GenoToul (Toulouse, France) for analysis purposes. A first quality control consisted in the visual inspection of the different graphs produced by fastqc (http://www.bioinformatics.babraham.ac.uk/projects/fastqc/), as well as in the alignment of the 
143

reads against classical contaminants such as Escherichia coli, yeast and phages. All the raw reads which passed this quality control were aligned to the CyHV-3 J strain genome (accession number AP008984) using BWA MEM (version bwa-0.7.5a) (Li \& Durbin 2009). Low quality alignments, i.e. reads aligning more than once or with poor sequencing quality inducing alignment quality lower than 30, were filtered out. The read depth was computed for the reference J-strain genome using Samtools (version 1.1) (Li et al. 2009). Variant calling was performed on the filtered alignments using the Genome Analyses Tool Kit (GATK, version 3.3.0) (DePristo et al. 2011), which improves the variant calling process through read realignment and base quality recalibration. PCR duplicates were filtered out using picard tools MarkDuplicates (version 1.88). Variations were thus obtained for single nucleotide polymorphisms (SNPs), insertions and deletions (indels) in VCF format files, using a Q20 filter. Both mapping and variant calling results were manually checked using the Integrative Genomics Viewer (IGV, version 2.3) (Thorvaldsdottir et al. 2013). Finally, regions showing high coverage were checked for contamination using blastn with standard settings (Altschul et al. 1997) against the NCBI nucleotide collection (nr/nt) database. Figures representing the sequencing depth and the number of small nucleotide variants were created with the $\mathrm{R}$ packages ggplot 2 and limma, respectively.

A de novo assembly of the reads was concomitantly performed for each sample using ABySS, and Cap3 programs. Briefly, fastq files were trimmed with Fastq trimmer (version 1.0.0) (Blankenberg et al. 2010) using the default parameters and the resulting reads were assembled in paired-end by ABySS v1.5.0 (Simpson et al. 2009). The obtained contigs were subjected to a new assembly using Cap3 (Huang \& Madan 1999), and resulting assemblies were mapped on a CyHV-3 reference genome (strain U, accession number DQ657948) using Bowtie 2 (version 2.2.1) program (Langmead et al. 2009). Consensus sequences were generated from these assemblies, in which indels and SNPs were validated when more than $50 \%$ of the reads harbored the variation.

\section{Genome comparisons}

Read alignments were also subjected to a genetic distance analysis. Variant calling files of individual specimens were compared through an identity-by-state calculation using PLINK, with the distance option (Chang et al. 2015). Reads were artificially created for the 3 reference genomes $\mathrm{U}, \mathrm{I}$ and $\mathrm{J}$ in order to include them in the analyses. For this purpose, 100-base reads were artificially produced every 5 bases along the reference genome with a simple python script. These reads were then randomly shuffled to form a set of reads with an even 20x depth, using samtools faidx (version 0.1.19). Then a hierarchical cluster analysis was computed with a complete-linkage clustering method, using the standard parameters, and dendrograms were plotted with the hclust $\mathrm{R}$ package. A visual representation of the distribution of nucleotide variants (SNPs + indels) was also created with Circos software (Krzywinski et al. 2009), using the genome of CyHV-3 J strain as reference. For clarity, only the genomes of PoB3_1, $\mathrm{J} 1 \_101110$ and I09_2i3 were represented.

The seven consensus sequences were aligned together with the 3 already sequenced genomes ( $\mathrm{J}$, $\mathrm{U}$ and I strains) using the online Multiple alignment program for amino acid or nucleotide sequences (Mafft program version 7, http://mafft.cbrc.jp/alignment/server/). The resulting alignments were visualized and manually corrected with GeneDoc version 2.7 (Nicholas et al. 1997). Then the evolutionary history was inferred by the Neighbor-Joining method (Saitou \& Nei 1987), and evolutionary distances were computed with MEGA6 (Tamura et al. 2013), using 
189

190

191

192

193

194

195

196

197

198

199

200

201

202

203

204

205

206

207

208

209

210

211

212

213

214

215

216

217

218

219

220

221

222

223

224

225

226

227

228

229

230

231

232

233

234

the Maximum Composite Likelihood method (Tamura et al. 2004). All positions containing gaps and missing data were eliminated.

Nucleotide sequence accession numbers

The raw sequences (fastq files) of the whole project are available under the SRP study accession SRP059764 (http://trace.ncbi.nlm.nih.gov/Traces/sra/?study=SRP059764). It is organized in 7 biosamples and 10 experiments, as shown in Table 1. Sequences corresponding to the 7 consensus genomes have also been submitted to GenBank and can be accessed under the following accession numbers: KX544842, KX544843, KX544844, KX544845, KX544846, KX544847 and KX544848 (for PoB3, J2_101110, CB4_181110, J1_101110, I_09_2i3, I_10_3 and PP3_070411, respectively).

\section{Results}

Viral genomic DNA enrichment: read mapping, sequencing depth and genome coverage

A total of $316,007,054$ reads of $100 \mathrm{bp}$ were obtained for the ten libraries, resulting in about 31.6 $\mathrm{Gb}$ of sequence data. The number of reads per library ranged from 9,163,662 (PoB3_3) to 56,046,694 (I_09-2i3) (Table 2). The rate of enrichment, reflected by the proportion of mapped reads, apparently correlated with the initial viral load, as it tended to increase concomitantly. For specimens with viral loads $>10^{6}$ copies, the proportion of mapped reads (after duplicate removal) was comprised between 43.99\% (PP3_070411) and 50.70\% (CB4_181110). Conversely, for PoB3, it varied between $3.22 \%$ and $7.22 \%$ of the total number of reads, depending on the assay format. Removal of duplicate sequences generally led to a significant reduction in the proportion of reads that could be mapped on the CyHV-3 reference genome (strain J). Sequence depth along this reference genome was variable both across and within specimens. It was $>2000 x$ on average for all specimens with an estimated number of viral copies $>10^{5}$, and $>200 \mathrm{x}$ for all other specimens (Table 2 and Supplementary figure 1). For most samples, there was an over representation of reads at positions 226,373 - 226,831 and 228,015 228,163 (according to KHV-J coordinates, GenBank \# AP008984). A Blast search revealed that these regions shared $97 \%$ and $94 \%$ identity with sequences of Cyprinus carpio genome (GenBank \# LN591727 and LN591823, respectively). However, this over representation was attenuated in samples with high initial viral loads (J1_101110, CB4_181110 and PP3_070411). A higher coverage (x30) was also noticed for J2_101110 specimen in the region corresponding to positions 45,500 - 47,700; yet this portion of $\overline{C y H V}-3$ genome did not return any significant blast hit. However, since the genome of Cyprinus carpio is not yet complete, it cannot be ruled out that these sequences belong to the carp genome. In spite of these slight differences, genomes could be recovered in their totality (or near-totality) from all samples, since coverage along the reference sequence was above $99.9 \%$, with the exception of PoB3_2 for which coverage was slightly lower $(99.05 \%)$.

\section{Comparison of PoB3 sequences according to the assay format}

Comparison of PoB3 in the different situations suggests that pre-capture multiplexing had an influence on the number of raw reads: the number of obtained sequences was highest when PoB3 was enriched separately, lowest when mixed with specimens with high viral loads (PoB3_3) and intermediate when mixed with specimens displaying lower viral loads (PoB3_4). The proportion of mapped reads (before duplicate removal) followed the same trend, with the noticeable 
235

exception of PoB3_2 (89.40\%) (Table 2 and Supplementary Figure 2). However, this high proportion of mapped reads obtained with the double-capture protocol (PoB3_2) dropped to $4.59 \%$ after sequence duplicate removal, suggesting that the majority of these sequences corresponded to duplicates. Even though the mean depth values of the 4 PoB3 samples were comparable, their distribution along the genome, however, displayed marked differences: whereas sequence representation was the steadiest along the genome for PoB3_1, it was uneven for PoB3_2, and in a lesser extent for PoB3_3 and PoB3_4 (figure 1).

When compared to KHV-J reference strain, the number of variations (SNP + indel) observed for the 4 PoB3 samples varied from 423 (PoB3_1) to 457 (PoB3_3). Among these variations, 335 were shared by all 4 PoB3 samples (Figure 2). PoB3_3 displayed the highest number of 'unique' variations (75), representing $\sim 16 \%$ of the total variations recorded for this sample. Examination of these 75 positions revealed that they all were 'heterozygous', i.e. they consisted in a mix of two haplotypes, including that of the reference genome. Fifty five of these variations were shared with at least one of the 3 other co-enriched samples (J1-101110, CB4-181110, PP3_070411), and 10 additional ones were located in the region that matches with the host genome (between positions 226,373 and 228,163 according to KHV-J coordinates). A distance analysis including the four PoB3 replicates (Supplementary Figure 3) indicated that the impact of these differences on the general phylogeny was very limited.

\section{Inter- and intra-specimen variations}

Using KHV-J as reference, sequence identity ranged from $99.85 \%$ to $99.97 \%$. The most divergent sequence was that of PoB3. This corresponded to approximately 425 sites that varied from the reference KHV-J sequence. The proportion of single nucleotide polymorphisms (SNP) and insertions/deletions (indel) was nearly 50\%-50\% for all samples (Table 3). As can be visualized on figure 4 , variations were distributed across the entire genome, with no particular hotspot of variability. In addition to the differences between specimens, sequence variability also occurred within specimens. Indeed, the proportion of varying sites harboring more than one allele (using the GATK criteria) was above 30\% for all specimens (Table 3). If some of this intra-specimen variability may probably be attributed to experimental biases (see discussion below), it nonetheless suggests the presence of multiple genotypes within a single specimen. Because of this intra-specimen sequence heterogeneity, distance analysis between specimens was realized directly from vcf files, using the PLINK software. Results indicated that PoB3 belonged to the European lineage, whereas all Indonesian specimens belonged to the Asian one (Figure 3). Multiple sequence alignment realized on the seven generated consensus genomes and the three reference CyHV-3 genomes led to the same result (Supplementary Figure 4).

\section{Discussion}

Concentration of viral particles through cell culture propagation (providing a suitable cell line is available for the investigated virus) is not always successful and requires days to obtain sufficient viral titers. Using targeted sequence capture prior to sequencing enabled here to recover ten complete (or near-complete) 295-kbp genomes in one single run of sequencing, directly from infected tissues, and with mean read depth varying from $219 x$ to $9179 x$. For comparison, Liu et al. used 24 genomic DNA libraries of Epstein Barr virus (EBV) to recover one full genome from a tumor tissue with an average read depth of 17x (Liu et al. 2011). This illustrates the potential of TGE to overcome the high discrepancy in the proportions of cellular 
281

282

283

284

285

286

287

288

289

290

291

292

293

294

295

296

297

298

299

300

301

302

303

304

305

306

307

308

309

310

311

312

313

314

315

316

317

318

319

320

321

322

323

324

325

326

and viral genomic DNA in raw samples obtained from infected tissues. Sample multiplexing is now routinely employed in next-generation sequencing, through the use of specific barcodes (Parameswaran et al. 2007). However, there are a limited number of studies describing precapture multiplexing, though it may improve both efficiency and cost-effectiveness of TGE (Shearer et al. 2012). In the present case, a rough estimation indicated that $4 x$ multiplexing resulted in a drop of the per sample price from $\sim 1600 €$ to $\sim 450 €$ (excluding sequencing costs).

Although there are no replicates to allow for statistical confirmation, the results obtained on PoB3 samples revealed some interesting differences. First, the double-capture protocol resulted in a higher number of uncovered positions, suggesting that the repeated amplifications have generated a bias in sequence representation. Though this aspect is not well documented in the literature, it has been shown, however, that the time of barcode incorporation during the library preparation and its distance from the priming site, as well as the PCR extension temperature, were potential sources of bias (López-Barragán et al. 2011; Van Nieuwerburgh et al. 2011). Second, while pre-capture multiplexing entailed a decrease in the number of reads, it did not compromise the sequencing depth or the coverage along the genome. Third, there were slight differences in the number of variations found between the four PoB3 samples and KHV-J reference genome, with PoB3_3 displaying the highest number of 'unshared' variations, i.e. variations that were not recorded in the other PoB3 samples. It is interesting to note that the 75 variations that were specific to $\mathrm{PoB} 3$ _ 3 were all heterozygous, and that the majority of them were shared by at least one of its co-enriched counterparts. Of these 75 variants, only 10 were located in the region that shares a high sequence identity with the carp reference genome. This evidence is likely indicative of low level cross-contamination with co-enriched samples. Comparison with PoB3_4, for which the 13 specific variations were also heterozygous and shared by at least one of the multiplexed samples, suggests that such a contamination would be favored by the difference in the initial viral loads of the multiplexed samples. For this reason, it seems advisable to multiplex samples with comparable viral loads.

It is generally acknowledged that errors during the mapping of cluster coordinates may result in $1-2 \%$ of bias in assignment of reads to sample during demultiplexing (Krueger et al. 2011). Considering the great difference in the sequencing depth between PoB3 samples and the other specimens, such a "contamination" could explain these faint differences between PoB3 samples. Moreover, an additional source of inaccuracy may also emanate from amplification and/or sequencing errors. In order to evaluate the contribution of pre-capture multiplexing on these observed differences, a specimen with a high viral load $\left(1.5 \times 10^{8}\right.$ viral copies $)$ was passaged on CCB (common carp brain) cells and sequenced: 1) after TGE in a 4-plex format with specimens of comparable viral loads; 2) without enrichment. Comparison of the resulting sequences revealed only 18 differences over the $295-\mathrm{kbp}$ genomes between the enriched and non-enriched samples (data concerning this specimen and additional ones will be published separately). This result brings additional evidence that pre-capture multiplexing does not induce any significant bias as long as the multiplexed specimens have comparable viral loads, and/or as long as the initial viral amount is sufficient to generate elevated read depth $(>1000 \mathrm{x})$. In any case, the differences between the four PoB3 samples represented nearly 0.004 to $0.025 \%$ of the genome, i.e. 4-25 differences every $100 \mathrm{~kb}$. This error rate appears acceptable, especially if we consider the low number of viral copies that were initially contained in this specimen.

Considering the relatively low viral load of J2_101110 specimen, we can also expect that a number of observed variations may constitute 'false callings'. This can be anticipated by i) the total number of detected variations (171), which is slightly higher than in the other Indonesian 
specimens (100-126); and ii) the higher proportion of heterozygous variations compared with the other Indonesian specimens. Even though the proportion of heterozygous positions is certainly over-estimated, especially in samples that were multiplexed prior to TGE, such a high rate of intra-specimen variability confirms previous findings that carp are frequently infected with a mix of CyHV-3 genotypes (Sunarto et al. 2011). The way such mixed infections arise in their host still remains speculative, as well as their ecological and evolutionary advantage. Now, the possibility to access full viral genomes from their host will undoubtedly contribute to a better understanding of CyHV-3 mixed infections.

The genomic distance analysis indicated that PoB3 specimen belonged to the European lineage, whereas the Indonesian samples fell in the Asian lineage. However, as was formerly shown (Aoki et al. 2007; Li et al. 2015), the divergence remains very low at the genome scale. The first KHVD outbreak reported in Indonesia dates back to 2002 (Sunarto et al. 2002). Since then, the disease rapidly spread throughout the whole country, especially in the West Java region (Sunarto et al. 2005). The use of 12 VNTR (variable number of tandem repeat) markers on the six investigated CyHV-3 Indonesian specimens revealed an important diversity of genotypes, which is not reflected at the genome scale (Avarre et al. 2012). This is not surprising, as tandem repeats evolve faster than any other part of a genome. Whereas some tandem repeats do not carry any adaptive value, others, however, allow functional diversification and may be involved in pathogenic pathways (Mrázek et al. 2007; Treangen et al. 2009). Therefore, full genomic comparisons will probably be more suitable to trace back outbreaks and to understand the transmission routes of CyHV-3, as they will also enable the study of the variations of short tandem repeats contained in the genomes of CyHV-3.

\section{Conclusion}

This TGE strategy enabled the successful recovery of full-length genomes from samples containing as little as 5000 viral copies. Though pre-capture multiplexing of samples with low viral loads is prone to generate false variants, it dramatically reduces the cost of an experiment. From our results, we would advise to pre-capture multiplex only samples with an initial number of viral copies above $10^{5}$, and to multiplex samples with comparable loads whenever possible. This TEG strategy will enable to analyze the genomic diversity of CyHV-3 and to study the dynamics of in vivo infections, by accessing the possible heterogeneity in genome populations through the analysis of the nucleotide composition at each position along the genome.

\section{Acknowledgement}

This is publication IRD-DIVA-ISEM 2016-162. 


\section{References}

Altschul SF, Madden TL, Schäffer AA, Zhang J, Zhang Z, Miller W, and Lipman DJ. 1997. Gapped BLAST and PSI-BLAST: a new generation of protein database search programs. Nucleic Acids Research 25:3389-3402.

Aoki T, Hirono I, Kurokawa K, Fukuda H, Nahary R, Eldar A, Davison AJ, Waltzek TB, Bercovier H, and Hedrick RP. 2007. Genome sequences of three koi herpesvirus isolates representing the expanding distribution of an emerging disease threatening koi and common carp worldwide. Journal of Virology 81:5058-5065.

Avarre JC, Madeira JP, Santika A, Zainun Z, Baud M, Cabon J, Caruso D, Castric J, Bigarre L, Engelsma M, and Maskur M. 2011. Investigation of Cyprinid herpesvirus-3 genetic diversity by a multi-locus variable number of tandem repeats analysis. $J$ Virol Methods 173:320-327.

Avarre JC, Santika A, Bentenni A, Zainun Z, Madeira JP, Maskur M, Bigarré L, and Caruso D. 2012. Spatio-temporal analysis of Cyprinid herpesvirus 3 genetic diversity at a local scale. Journal of Fish Diseases 35:767-774.

Bigarré L, Baud M, Cabon J, Antychowicz J, Bergmann SM, Engelsma M, Pozet F, Reichert M, and Castric J. 2009. Differentiation between Cyprinid herpesvirus type-3 lineages using duplex PCR. Journal of Virological Methods 158:51-57.

Blankenberg D, Gordon A, Von Kuster G, Coraor N, Taylor J, and Nekrutenko A. 2010. Manipulation of FASTQ data with Galaxy. Bioinformatics 26:1783-1785.

Chang C, Chow C, Tellier L, Vattikuti S, Purcell S, and Lee J. 2015. Second-generation PLINK: rising to the challenge of larger and richer datasets. GigaScience 4:7.

Davison AJ. 2010. Herpesvirus systematics. Veterinary Microbiology 143:52-69.

Depledge DP, Palser AL, Watson SJ, Lai IY-C, Gray ER, Grant P, Kanda RK, Leproust E, Kellam P, and Breuer J. 2011. Specific Capture and Whole-Genome Sequencing of Viruses from Clinical Samples. PLoS ONE 6:e27805.

DePristo MA, Banks E, Poplin R, Garimella KV, Maguire JR, Hartl C, Philippakis AA, del Angel G, Rivas MA, Hanna M, McKenna A, Fennell TJ, Kernytsky AM, Sivachenko AY, Cibulskis K, Gabriel SB, Altshuler D, and Daly MJ. 2011. A framework for variation discovery and genotyping using next-generation DNA sequencing data. Nat Genet 43:491-498.

Hedrick RP, Gilad O, Yun S, Spangenberg JV, Marty GD, Nordhausen RW, Kebus MJ, Bercovier H, and Eldar A. 2000. A herpesvirus associated with mass mortality of juvenile and adult koi, a strain of common carp. Journal of Aquatic Animal Health 12:44-57.

Huang X, and Madan A. 1999. CAP3: A DNA Sequence Assembly Program. Genome Research 9:868-877.

King AMQ, Adams MJ, Carstens EB, and Lefkowitz EJ. 2011. Virus taxonomy: Ninth Report of the International Committee on Taxonomy of Viruses. San Diego: Elsevier Academic Press. p 1327.

Krueger F, Andrews SR, and Osborne CS. 2011. Large Scale Loss of Data in Low-Diversity Illumina Sequencing Libraries Can Be Recovered by Deferred Cluster Calling. PLoS ONE 6:e16607.

Krzywinski MI, Schein JE, Birol I, Connors J, Gascoyne R, Horsman D, Jones SJ, and Marra MA. 2009. Circos: An information aesthetic for comparative genomics. Genome Research. 
410

411

412

413

414

415

416

417

418

419

420

421

422

423

424

425

426

427

428

429

430

431

432

433

434

435

436

437

438

439

440

441

442

443

444

445

446

447

448

449

450

451

452

453

454

455

Kurita J, Yuasa K, Ito T, Sano M, Hedrick RP, Engelsma MY, Haenen OLM, Sunarto A, Kholidin EB, Chou HY, Tung MC, de la Pena L, Lio-Po G, Tu C, Way K, and Iida T. 2009. Molecular Epidemiology of Koi Herpesvirus. Fish Pathology 44:59-66.

Langmead B, Trapnell C, Pop M, and Salzberg S. 2009. Ultrafast and memory-efficient alignment of short DNA sequences to the human genome. Genome Biology 10:R25.

Li H, and Durbin R. 2009. Fast and accurate short read alignment with Burrows-Wheeler transform. Bioinformatics 25:1754-1760.

Li H, Handsaker B, Wysoker A, Fennell T, Ruan J, Homer N, Marth G, Abecasis G, Durbin R, and Genome Project Data Processing S. 2009. The Sequence Alignment/Map format and SAMtools. Bioinformatics 25:2078-2079.

Li W, Lee X, Weng S, He J, and Dong C. 2015. Whole-genome sequence of a novel Chinese cyprinid herpesvirus 3 isolate reveals the existence of a distinct European genotype in East Asia. Veterinary Microbiology 175:185-194.

Liu P, Fang X, Feng Z, Guo YM, Peng RJ, Liu T, Huang Z, Feng Y, Sun X, Xiong Z, Guo X, Pang SS, Wang B, Lv X, Feng FT, Li DJ, Chen LZ, Feng QS, Huang WL, Zeng MS, Bei JX, Zhang Y, and Zeng YX. 2011. Direct sequencing and characterization of a clinical isolate of Epstein-Barr virus from nasopharyngeal carcinoma tissue by using nextgeneration sequencing technology. J Virol 85:11291-11299.

López-Barragán MJ, Quiñones M, Cui K, Lemieux J, Zhao K, and Su X-z. 2011. Effect of PCR extension temperature on high-throughput sequencing. Molecular and Biochemical Parasitology 176:64-67.

Mariette J, Escudie F, Allias N, Salin G, Noirot C, Thomas S, and Klopp C. 2012. NG6: Integrated next generation sequencing storage and processing environment. Bmc Genomics 13:462.

Mertes F, ElSharawy A, Sauer S, van Helvoort J, van der Zaag PJ, Franke A, Nilsson M, Lehrach H, and Brookes AJ. 2011. Targeted enrichment of genomic DNA regions for next-generation sequencing. Briefings in Functional Genomics 10:374-386.

Mrázek J, Guo X, and Shah A. 2007. Simple sequence repeats in prokaryotic genomes. Proceedings of the National Academy of Sciences 104:8472-8477.

Nicholas KB, Nicholas HBJ, and Deerfield DW. 1997. GeneDoc: Analysis and visualization of genetic variation. EMBNEWNEWS 4:14.

Parameswaran P, Jalili R, Tao L, Shokralla S, Gharizadeh B, Ronaghi M, and Fire AZ. 2007. A pyrosequencing-tailored nucleotide barcode design unveils opportunities for large-scale sample multiplexing. Nucleic Acids Research 35:e130.

Pokorova D, Vesely T, Piackova V, Reschova S, and Hulova J. 2005. Current knowledge on koi herpesvirus (KHV): a review. Veterinarni Medicina 50:139-147.

Saitou N, and Nei M. 1987. The neighbor-joining method: a new method for reconstructing phylogenetic trees. Mol Biol Evol 4:406-425.

Shearer AE, Hildebrand MS, Ravi H, Joshi S, Guiffre AC, Novak B, Happe S, Le Proust EM, and Smith RJH. 2012. Pre-capture multiplexing improves efficiency and costeffectiveness of targeted genomic enrichment. Bmc Genomics 13:8.

Simpson JT, Wong K, Jackman SD, Schein JE, Jones SJM, and Birol İ. 2009. ABySS: A parallel assembler for short read sequence data. Genome Research 19:1117-1123.

Sunarto A, McColl KA, Crane MS, Sumiati T, Hyatt AD, Barnes AC, and Walker PJ. 2011. Isolation and characterization of koi herpesvirus (KHV) from Indonesia: identification of a new genetic lineage. Journal of Fish Diseases 34:87-101. 
456

457

458

459

460

461

462

463

464

465

466

467

468

469

470

471

472

473

474

475

476

477

478

479

480

481

482

483

484

485

Sunarto A, Rukyani A, and Itami T. 2005. Indonesian Experience on the Outbreak of Koi Herpesvirus in Koi and Carp

(Cyprinus carpio). Bull Fish Res Agen Supplement No. 2,:15-21.

Sunarto A, Taukhid, Rukyani A, Koesharyani I, Supriyadi H, Huminto H, Agungpriyono DR, Pasaribu FH, Widodo, Herdikiawan D, Rukmono D, and Prayitno SB. 2002. Field investigations on a serious disease outbreak among koi and common carp (Cyprinus carpio) in Indonesia. 5th Symposium on Diseases in Asian Aquaculture. Gold Cost, Australia.

Tamura K, Nei M, and Kumar S. 2004. Prospects for inferring very large phylogenies by using the neighbor-joining method. Proceedings of the National Academy of Sciences of the United States of America 101:11030-11035.

Tamura K, Stecher G, Peterson D, Filipski A, and Kumar S. 2013. MEGA6: Molecular Evolutionary Genetics Analysis version 6.0. Mol Biol Evol 30:2725-2729.

Thorvaldsdottir H, Robinson JT, and Mesirov JP. 2013. Integrative Genomics Viewer (IGV): high-performance genomics data visualization and exploration. Brief Bioinform 14:178192.

Treangen TJ, Abraham AL, Touchon M, and Rocha EP. 2009. Genesis, effects and fates of repeats in prokaryotic genomes. FEMS Microbiol Rev 33:539-571.

Van Nieuwerburgh F, Soetaert S, Podshivalova K, Ay-Lin Wang E, Schaffer L, Deforce D, Salomon DR, Head SR, and Ordoukhanian P. 2011. Quantitative Bias in Illumina TruSeq and a Novel Post Amplification Barcoding Strategy for Multiplexed DNA and Small RNA Deep Sequencing. PLoS ONE 6:e26969.

Waltzek TB, Kelley GO, Alfaro ME, Kurobe T, Davison AJ, and Hedrick RP. 2009. Phylogenetic relationships in the family Alloherpesviridae. Diseases of Aquatic Organisms 84:179-194.

Waltzek TB, Kelley GO, Stone DM, Way K, Hanson L, Fukuda H, Hirono I, Aoki T, Davison AJ, and Hedrick RP. 2005. Koi herpesvirus represents a third cyprinid herpesvirus (CyHV-3) in the family Herpesviridae. Journal of General Virology 86:1659-1667.

Yuasa K, Sano M, Kurita J, Ito T, and Iida T. 2005. Improvement of a PCR method with the Sph 1-5 primer set for the detection of koi herpesvirus (KHV). Fish Pathology 40:37-39. 
Table 1 Assay design for CyHV-3 sequence capture

\begin{tabular}{llllll}
\hline Assay format & $\begin{array}{l}\text { Specimen } \\
\text { name }\end{array}$ & $\begin{array}{l}\text { Estimated } \\
\text { number of } \\
\text { viral copies }\end{array}$ & $\begin{array}{l}\text { Country and year } \\
\text { of collection }\end{array}$ & Reference & $\begin{array}{l}\text { Experiment } \\
\text { accession \# }\end{array}$ \\
\hline Simplex & PoB3_1 & $4.8 \mathrm{E}+3$ & Poland, 2013 & This study & SRX1071741 \\
\hline $\begin{array}{l}\text { Simplex, double } \\
\text { capture }\end{array}$ & PoB3_2 & $4.8 \mathrm{E}+3$ & Poland, 2013 & This study & SRX1071742 \\
\hline & PoB3_3 & $4.8 \mathrm{E}+3$ & Poland, 2013 & This study & SRX1071743 \\
4-plex & J1_101110 & $1.5 \mathrm{E}+7$ & Indonesia, 2010 & Avarre et al, 2012 & SRX1071745 \\
& CB4_181110 & $1.9 \mathrm{E}+7$ & Indonesia, 2010 & Avarre et al, 2012 & SRX1071746 \\
& PP3_070411 & $5.7 \mathrm{E}+6$ & Indonesia, 2011 & Avarre et al, 2012 & SRX1071747 \\
\hline & PoB3_4 & $4.8 \mathrm{E}+3$ & Poland, 2013 & This study & SRX1071744 \\
4-plex & I_10-3 & $1.5 \mathrm{E}+5$ & $\begin{array}{l}\text { Indonesia, 2010 } \\
\text { Indonesia, 2009 }\end{array}$ & Avarre et al, 2011 & SRX1071748 \\
& I_09-2i3 & $1.5 \mathrm{E}+5$ & Indonesia, 2010 & Avarre et al, 2012 & SRX1071750 \\
\hline
\end{tabular}

${ }^{a}$ Before capture of viral DNA 
Table 2 Main features of enrichment and sequencing results

\begin{tabular}{|c|c|c|c|c|c|c|c|}
\hline $\begin{array}{l}\text { Specimen } \\
\text { name }\end{array}$ & \# raw reads & $\begin{array}{l}\% \\
\text { mapped } \\
\text { reads }^{\mathrm{a}}\end{array}$ & $\begin{array}{l}\text { \% mapped } \\
\text { reads after } \\
\text { duplicate } \\
\text { removal }^{\mathrm{a}}\end{array}$ & $\begin{array}{l}\text { Mean depth } \\
(\mathrm{x})^{\mathrm{a}, \mathrm{b}}\end{array}$ & $\begin{array}{l}\text { Coverage } \\
\text { along the } \\
\text { reference } \\
\text { genome }(\%)^{\mathrm{a}}\end{array}$ & $\begin{array}{l}\text { Number of } \\
\text { uncovered } \\
\text { positions }^{\mathrm{a}}\end{array}$ & $\begin{array}{l}\text { Estimated } \\
\text { number of } \\
\text { viral copies }\end{array}$ \\
\hline PoB3_1 & 22852739 & 28.73 & 3.22 & 221 & 99.99 & 32 & $4.8 \mathrm{E}+3$ \\
\hline PoB3_2 & 28611191 & 89.40 & 4.59 & 409 & 99.05 & 2600 & $4.8 \mathrm{E}+3$ \\
\hline PoB3_3 & 9163662 & 16.67 & 7.22 & 219 & 99.92 & 218 & $4.8 \mathrm{E}+3$ \\
\hline $\mathrm{J} 1 \_101110$ & 32892125 & 80.47 & 49.66 & 5931 & 99.94 & 168 & $1.5 \mathrm{E}+7$ \\
\hline CB4_181110 & 49788380 & 88.81 & 50.70 & 9179 & 99.99 & 29 & $1.9 \mathrm{E}+7$ \\
\hline PP3_070411 & 42335046 & 76.32 & 43.99 & 6741 & 99.96 & 115 & $5.7 \mathrm{E}+6$ \\
\hline PoB3_4 & 16182800 & 33.78 & 4.90 & 246 & 100 & 0 & $4.8 \mathrm{E}+3$ \\
\hline I $10 \overline{3}$ & 44364454 & 67.84 & 16.46 & 2601 & 100 & 0 & $1.5 \mathrm{E}+5$ \\
\hline I_09_2i3 & 56046694 & 74.00 & 19.87 & 4015 & 100 & 0 & $1.5 \mathrm{E}+5$ \\
\hline $\mathrm{J} 2 \_101110$ & 14067420 & 31.61 & 5.26 & 235 & 99.90 & 272 & $8.8 \mathrm{E}+4$ \\
\hline
\end{tabular}

a Only Q30 sequences were considered

$492 \quad \mathrm{~b}$ after duplicate removal 
493 Table 3 Number of single nucleotide polymorphisms (SNPs) and insertions/deletions (indels) in 494 the different specimens compared to KHV-J

\begin{tabular}{lllll}
\hline Specimen & SNP & Indel & $\begin{array}{l}\text { \% small } \\
\text { nucleotide } \\
\text { variants }\end{array}$ & $\begin{array}{l}\text { a intra- } \\
\text { specimen } \\
\text { heterogeneous } \\
\text { sites }^{\text {a, b }}\end{array}$ \\
\hline PoB3_1 & 215 & 208 & 0.14 & 31.8 \\
PoB3_2 & 216 & 208 & 0.14 & 33.6 \\
PoB3_3 & 230 & 227 & 0.15 & 95.4 \\
J1-101110 & 47 & 66 & 0.04 & 31.3 \\
CB4-181110 & 43 & 57 & 0.03 & 31.3 \\
PP3_070411 & 66 & 60 & 0.04 & 42.5 \\
PoB3_4 & 213 & 211 & 0.14 & 93.0 \\
I10-3 & 72 & 53 & 0.04 & 72.1 \\
I_09-2i3 & 60 & 56 & 0.04 & 54.6 \\
J2_101110 & 94 & 77 & 0.06 & 81.9 \\
\hline
\end{tabular}

$495{ }^{a}$ Cut-off value set at $10 \%$ of the total number of reads at each specific position

$496{ }^{b}$ Excluding the terminal repeats

497 
498 Figure 1: Sequencing depth for PoB3 samples. (A) Boxplot representing the depth distribution in $499 \log 10$ of raw reads (red) and filtered reads (blue), i.e. after Q30 mapping quality trimming and 500 duplicate removal. (B) Per base sequencing depth along the KHV-J reference genome. The 501 figure was created with the R package ggplot2.

502

(A)

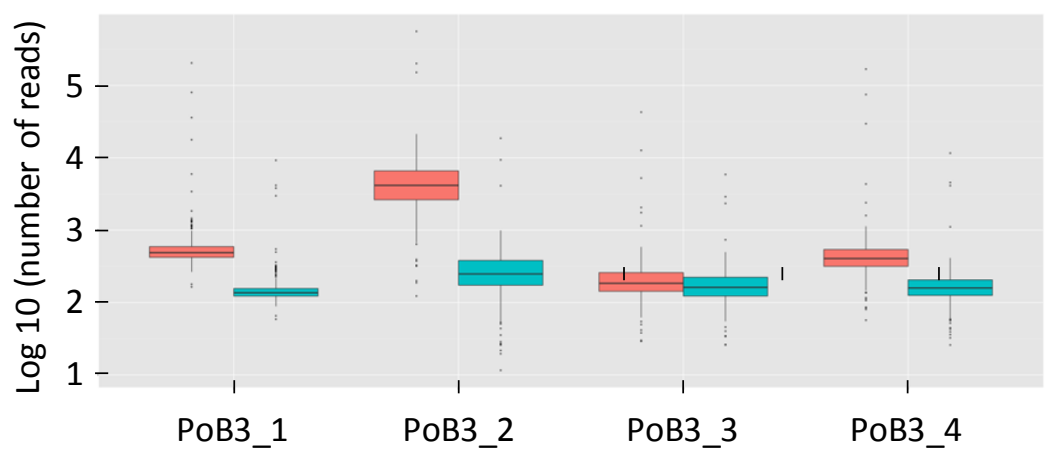

raw reads

after quality trimming

(B)

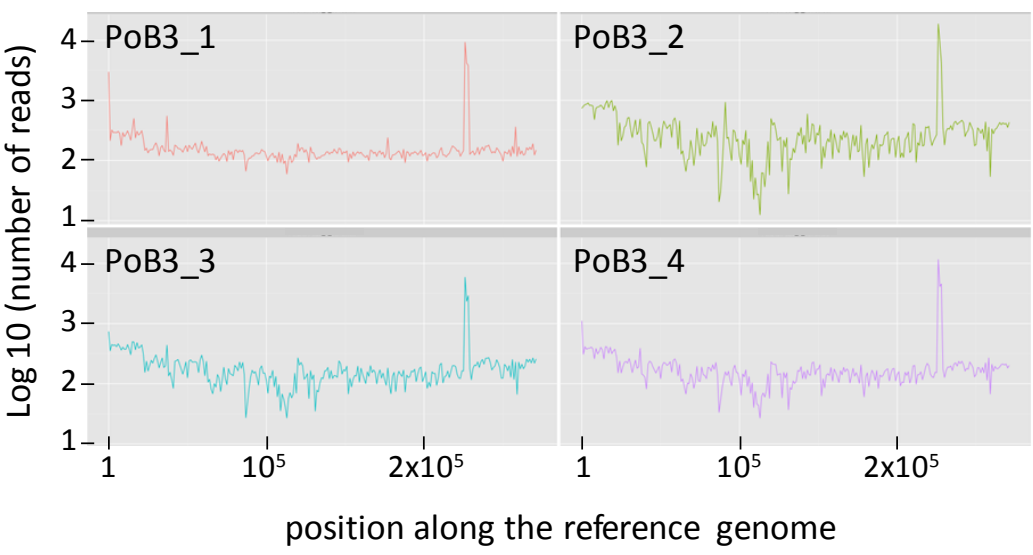


505 Figure 2: Comparison of the number of small nucleotide variants between PoB3 samples. 506 Intersections represent the variants that are shared between samples. This Venn diagram was 507 created with the $\mathrm{R}$ package limma.

508

509

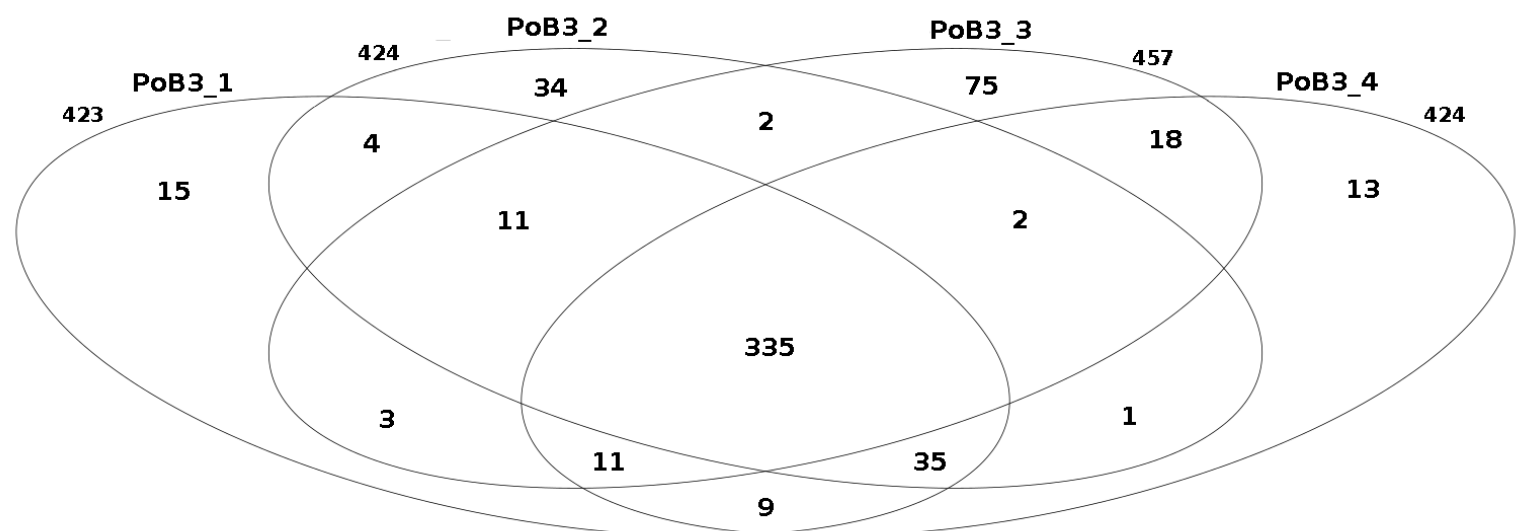


512 Figure 3: Genetic distances between CyHV-3 specimens. A distance matrix between samples 513 was first computed using an Identity By State (IBS) distance. Specimens were then grouped into 514 clusters using the $\mathrm{R}$ package hclust and the corresponding dendrogram plotted. The scale 515 represents the maximum IBS-distance between two elements of linked clusters (branches in the 516 tree).

517

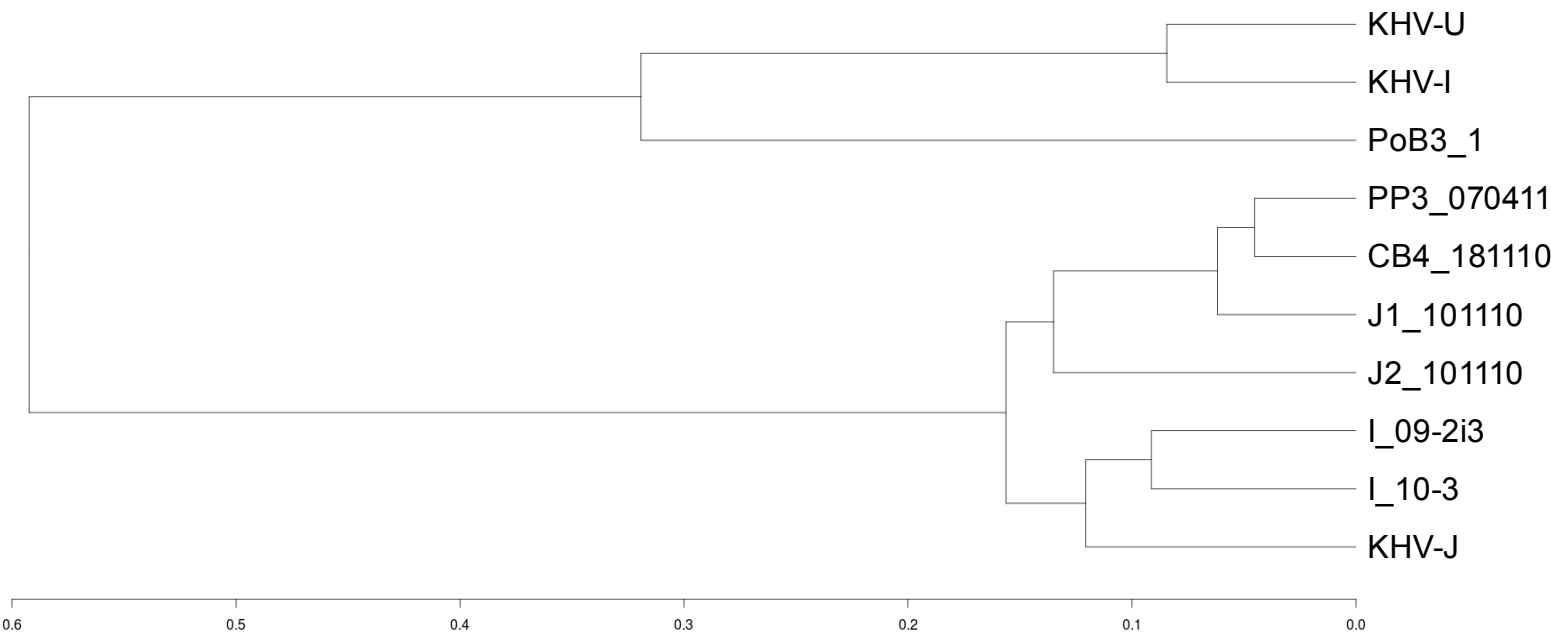


520 Figure 4: Distribution of nucleotide variations along CyHV-3 genomes. Numbers correspond to the genomic coordinates of $\mathrm{CyHV}-3 \mathrm{~J}$ strain, and the internal curves depict the small nucleotide variants (including SNPs and indels) found in the 3 genomes (PoB3_1, J1_101110 and I09_2i3) compared to CyHV-3 J strain. Peak height is proportional to the number of variations in a given region. A window size of $5 \mathrm{~kb}$ was used.

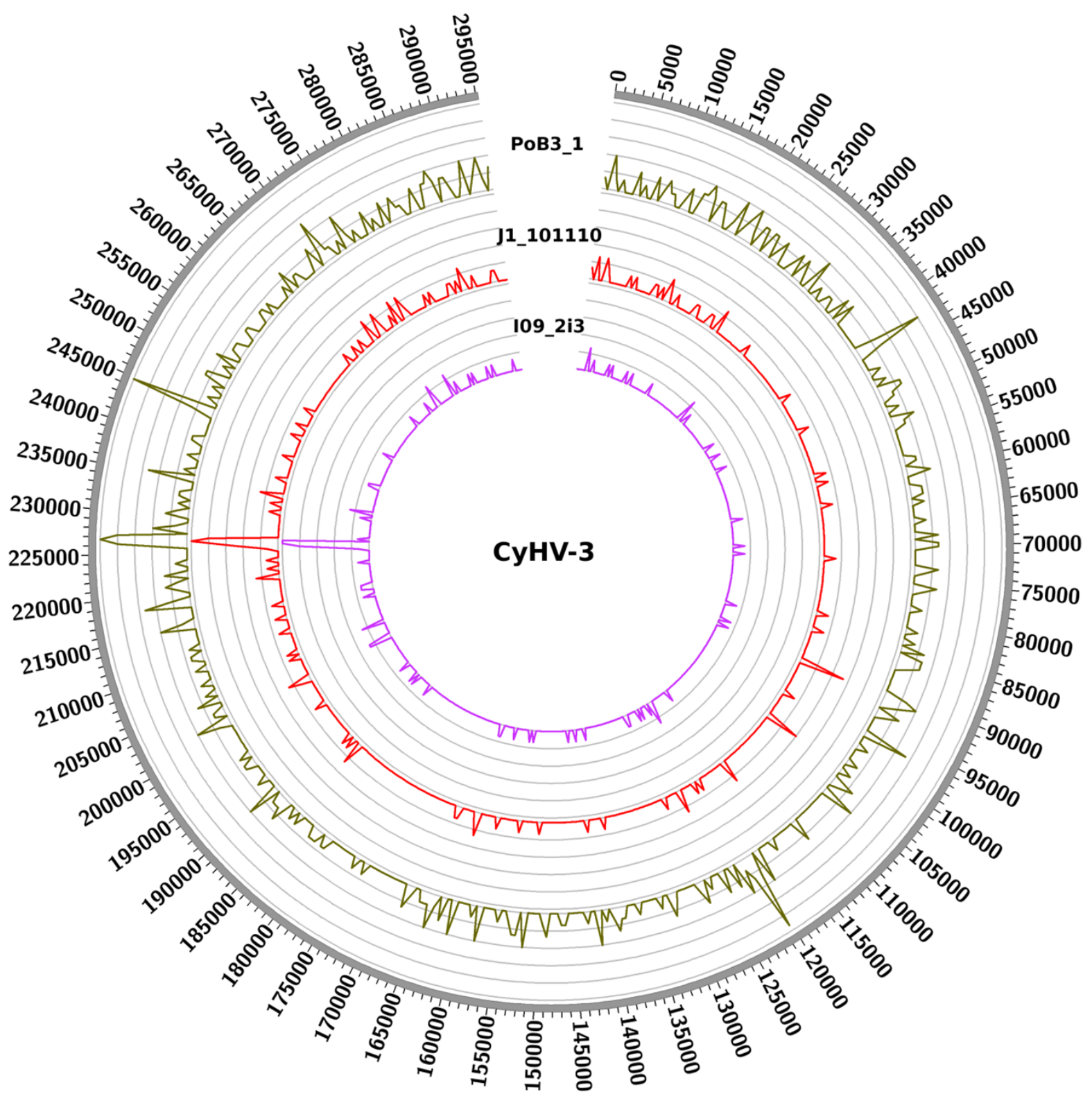

